\title{
Chapter 4. Primary kidney disease leading to dialysis therapy
}

- In Japan, the most frequent primary disease for dialysis is diabetic nephropathy, followed by chronic glomerulonephritis and nephrosclerosis as the third.

- Since the prevalence of metabolic syndrome, a risk factor for dialysis therapy, continues to increase, an urgent initiative against this syndrome is needed.

- The incidence of dialysis patients in Japan in 2007 was about 35,000 and is growing steadily. As of the end of 2007, the prevalence of dialysis patients was over 2,100 per million population, i.e., 1 per 464 persons is now on chronic dialysis (Fig. 4-1). Primary kidney diseases are diabetic nephropathy, chronic glomerulonephritis, and nephrosclerosis in descending order of incidence (Fig. 4-2). In 2007, dialysis was introduced because of diabetic nephropathy in $43.4 \%$ of the incident

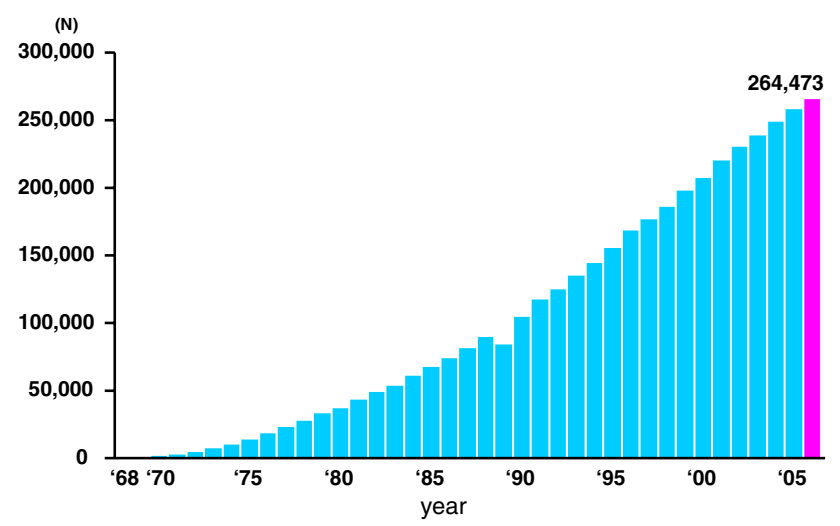

Fig. 4-1 Changes in the number of chronic dialysis patients in Japan. The number of chronic dialysis patients is steadily increasing about 10,000 a year. The data are quoted, with modification, from The Current Status of Chronic Dialysis Therapy in Our Country (as of 31 December, 2007) edited by The Japanese Society for Dialysis Therapy dialysis patients. Unidentified primary kidney disease is increasing steadily. The proportion of polycystic kidney is $2.3 \%$ and rapidly progressive glomerulonephritis $1.3 \%$, as shown in Table 4.

- Diabetic nephropathy overtook chronic glomerulonephritis as the leading cause for the introduction of dialysis in 1998. Since with metabolic syndrome, the risk of CKD is increasing more and more, an urgent initiative to prevent metabolic syndrome is required for the prevention of CKD.

- It is appreciated that the incidence of ESKD patients with chronic glomerulonephritis has been gradually decreasing in recent years (Fig. 4-2). There are various reasons for this decline. One reason is a decrease in infectious diseases that are related to the development of

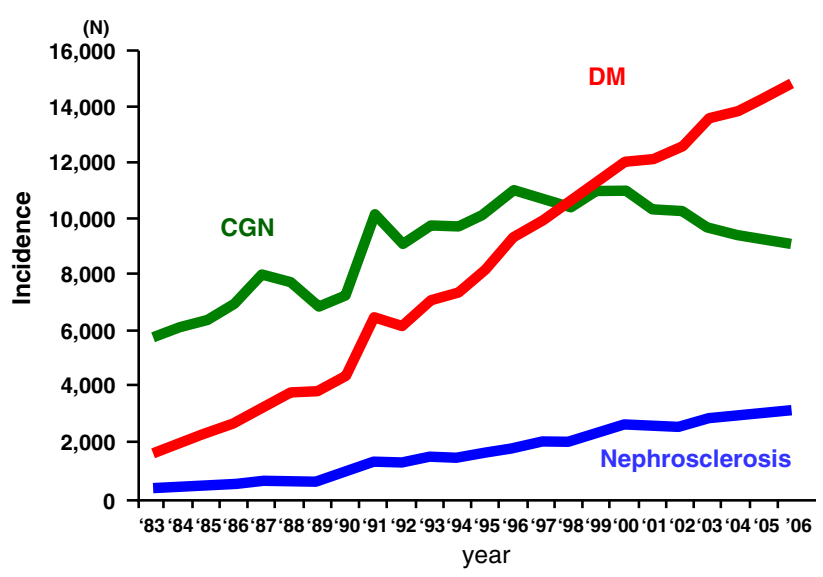

Fig. 4-2 Changes in the number of new dialysis patients in Japan (major primary kidney diseases). Diabetes has been the leading cause for the incidence of ESKD since 1998. Glomerulonephritis has been declining since 1997 but is still the second leading cause in Japan. Nephrosclerosis has been increasing in recent years and the third leading cause 
Table 4-1 Incident dialysis patients by kidney diseases

\begin{tabular}{|c|c|c|c|}
\hline Kidney disease & $\begin{array}{l}\text { Number of } \\
\text { patients }\end{array}$ & $\%$ & Rank \\
\hline DM nephropathy & 14,968 & 42.9 & 1 \\
\hline Chronic glomerulonephritis & 8,914 & 25.6 & 2 \\
\hline Unknown & 3,454 & 9.9 & 3 \\
\hline Nephrosclerosis & 3,262 & 9.4 & 4 \\
\hline Others & 903 & 2.6 & 5 \\
\hline Polycystic kidney disease & 827 & 2.4 & 6 \\
\hline RPGN & 421 & 1.2 & 7 \\
\hline Chronic pyelonephritis & 295 & 0.8 & 8 \\
\hline Malignant hypertension & 269 & 0.8 & 9 \\
\hline SLE & 268 & 0.8 & 10 \\
\hline Graft failure & 224 & 0.6 & 11 \\
\hline Amyloidosis & 168 & 0.5 & 12 \\
\hline Tumors in the genito-urinary system & 158 & 0.5 & 13 \\
\hline Unclassified GN & 149 & 0.4 & 14 \\
\hline Myeloma & 137 & 0.4 & 15 \\
\hline Obstructive uropathy & 128 & 0.4 & 16 \\
\hline Gouty kidney & 113 & 0.3 & 17 \\
\hline Genito-urinary stones & 75 & 0.2 & 18 \\
\hline Kidney malformation & 51 & 0.1 & 19 \\
\hline Pregnancy-related & 44 & 0.1 & 20 \\
\hline Congenital & 30 & 0.1 & 21 \\
\hline Genitourinary tuberculosis & 19 & 0.1 & 22 \\
\hline Total & 34,877 & 100.0 & \\
\hline
\end{tabular}

The data are quoted, with modification, from The Current Status of Chronic Dialysis Therapy in Our Country (as of 31 December, 2007) edited by The Japanese Society for Dialysis Therapy

nephritis or improvement of sanitation and social conditions. This is the case especially for the decreasing incidence of acute glomerulonephritis and membranoproliferative glomerulonephritis. Another reason is that chronic glomerulonephritis has been treated better with drug therapy, including "cocktail" therapy combining corticosteroid, immunosuppressants, and anticoagulation agents. Moreover, tonsillectomy with steroid pulse therapy has recently been reported to improve $\operatorname{IgA}$ nephropathy, the disease comprising more than $50 \%$ of

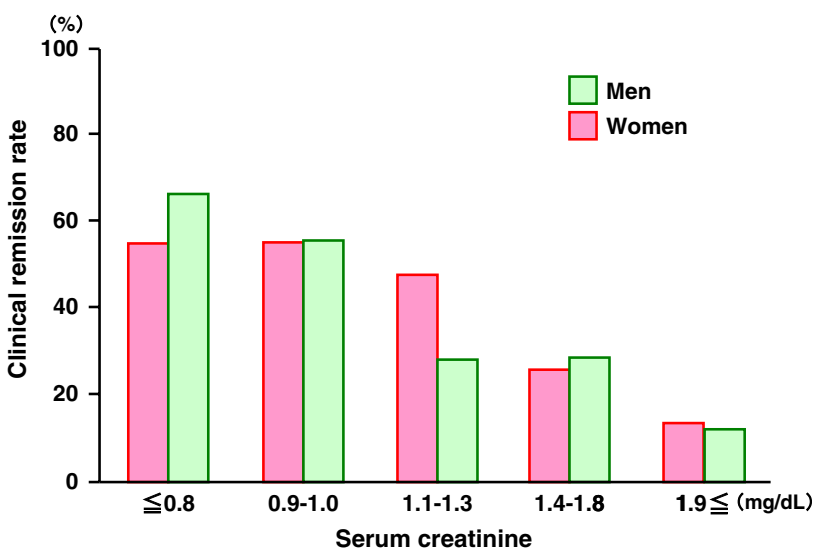

Fig. 4-3 Clinical remission rate of $\operatorname{IgA}$ nephropathy analyzed by serum creatinine at tonsillectomy followed by steroid pulse therapy. The data are quoted, with modification, from: Hotta $\mathrm{O}$ et al. (Am $\mathbf{J}$ Kidney Dis. 2001;38:736-743)

the cases of chronic glomerulonephritides in Japan (Fig. 4-3). In Fig. 4-3, clinical remission means the disappearance of both proteinuria and hematuria, and thus a remission case is expected to prevent progression to ESKD.

- The incidence of dialysis introduction because of nephrosclerosis, which is caused primarily by hypertension (including malignant hypertension), is still increasing and reached $10.0 \%$ in 2007 (Table 4-1). This increment is suspected to increase more in the future. Conceivably, hypertension is a risk factor for kidney dysfunction leading to dialysis in most of the kidney diseases such as diabetic nephropathy and chronic glomerulonephritis. Moreover, there is an increase in atherosclerosis due to metabolic syndrome and elderly populations. Atherosclerosis causes cerebrovascular disease as well as cardiovascular disease and further contributes to the development of CKD. Atherosclerosis-related nephropathy is rapidly increasing with an unfavorable prognosis and manifests as a variety of phenotypes, such as renal artery stenosis, renovascular hypertension, ischemic nephropathy, and cholesterol embolism. 